to evaluate accelerations and angles during COD movement, but so far there are no clear recommendations on specific metrics to be used.

Objective To evaluate the reliability and validity of inertial measurement unit (IMU) sensors to detect COD movement and aspects related to COD movement. To summarize the available evidence on how wearable IMUs are used to analyze COD movement in sports and exercise.

Design Scoping review. A systematic search was employed in MEDLINE (Ovid), CINAHL (EBSCO host), SPORTDiscus (EBSCO host), EMBASE and Cochrane Database of Systematic Reviews. A grey literature search was employed to locate nonpeer reviewed studies. The risk of bias of the studies evaluating validity and/or reliability was evaluated using the AXIS tool.

Setting Sport and exercise.

Participants Studies on sports related COD movements measured with IMUs.

Main Outcome Measurements Methodological quality of included studies on validity and reliability.

Results After screening 11,376 articles 47 studies remained, with eleven studies evaluating validity and/or reliability. Most of the studies were conducted with preplanned movements in the laboratory setting and participants were usually adult males. Varying sensor locations limits the ability to generalize these findings.

Conclusions There are promising results on validity and reliability of analysis of COD movement with IMUs, but the number of studies is small and the quality of the studies is limited. Studies using IMUs to evaluate COD movement can be improved with larger sample sizes and agreement on the metrics used and sensor placement. Future research should include on-field studies, where movements are unplanned and factors like speed and how opponent players affect the movements are included in analyses.

\section{THE INFLUENCE OF TRAINING VOLUME ON TRAINING AND MATCH INJURY RISK IN ELITE SCOTTISH RUGBY UNION PLAYERS}

\footnotetext{
${ }^{1}$ Cameron Paul, ${ }^{1}$ Tom Campbell, ${ }^{2}$ Stuart Yule, ${ }^{2}$ Jack Walsh, ${ }^{1}$ Russell Martindale, ${ }^{1,3}$ Debbie Palmer. 'School of Applied Sciences, Edinburgh Napier University, Edinburgh, UK; ${ }^{2}$ Scottish Rugby Union, Murrayfield, Edinburgh, UK; ${ }^{3}$ Institute for Sport, PE and Health Sciences, Moray House School of Education and Sport, University of Edinburgh, Edinburgh, UK
}

\subsection{6/bjsports-2021-IOC. 107}

Background Training volume has been shown to influence injury risk in elite Rugby Union players.

Objective To investigate the influence of training volume on injury risk in elite Scottish Rugby Union players.

Design A prospective, observational cohort study design was adopted to collect training volume (hours) and injury data (training and match time-loss injuries combined).

Setting Data were collected from Scottish Rugby Union's professional (Men's 15-a-side) teams (Men's International Squad; Glasgow Warriors and Edinburgh Rugby).

Patients (or Participants) Data were collected from 163 professional Rugby Union players over the 2017/18 and 2018/19 seasons.

Interventions (or Assessment of Risk Factors) Gym \& pitchbased training data were collected via team logs \& Global
Positioning System devices. Injury data were collected from the medical personnel associated with each team.

Main Outcome Measurements Derived workload measures were calculated. These included: the exponentially-weighted moving average acute: chronic workload ratio (ACWR); weekto-week change in volume, and 1- 2-, 3- and 4-week cumulative volumes. Workload measures were modelled against subsequent week injury using binary logistic regression analysis. Odds ratios (OR) were reported against a reference ('Verylow' workload) group.

Results Players spent a total of 58,044 hours training, and sustained 734 time-loss injuries. Compared to the reference category $(<0.50)$, an 'Intermediate-low' ACWR (0.75-1.00) had the lowest injury risk $(\mathrm{OR}=0.46)$. Contrary, an 'Intermediatehigh' (1.00-1.25), 'High' (1.25-1.50) and 'Very-high' (>1.50) ACWR significantly increased injury risk $(\mathrm{OR}=4.85,13.36$ and $15.70, p<0.001$, respectively). Injury risk was significantly increased for 'Intermediate-low' training volumes over 1-3 week cumulative periods, and 'Intermediate-high' volumes over 2-4 week cumulative periods. 'Very-high' volumes increased injury risk over 1-3 week cumulative periods. 'High' training volumes over 1-4 weeks and weekly change in volume were not associated with injury $(p>0.05)$.

Conclusions Increases in acute training volume beyond a player's current chronic status may increase injury risk. Minimising spikes in volume, whilst gradually acquiring high training volumes may be more protective against injury than intermediate and very high volumes.

\section{RELATIONSHIP BETWEEN READINESS INDICATORS, TRAINING LOAD AND FATIGUE IN COLLEGIATE FEMALE VOLLEYBALL ATHLETES}

${ }^{1,2}$ Javier Peña, ${ }^{3}$ Laurie Eisler, ${ }^{3}$ Carolyn O'Dwyer, ${ }^{1,2}$ Albert Altarriba-Bartés, ${ }^{1,2}$ Beatriz Gil, ${ }^{1,2}$ Clàudia Alba, ${ }^{3}$ Pierre Baudin. ${ }^{1}$ Sport and Physical Activity Studies Centre, University of Vic - Central University of Catalonia, Vic, Spain; ${ }^{2}$ Sport Performance Analysis Research Group, University of Vic - Central University of Catalonia, Vic, Spain; ${ }^{3}$ Faculty of Kinesiology, Sport, and Recreation, University of Alberta, Edmonton, Canada

\subsection{6/bjsports-2021-IOC.108}

Background Proper load monitoring can help to determine if athletes are adjusting properly to training loads, minimizing the risk of developing illnesses and injuries.

Objective The main objective of this study was to find relationships between internal and external load variables, and fatigue to enable a better understanding of specific adaptations.

Design An 8-week prospective observational cohort study with 213 observations.

Setting U Sports Canadian volleyball athletes.

Patients (or Participants) Six female volleyball athletes $(21 \pm 2$ years, $179.8 \pm 6.1 \mathrm{~cm}, 72 \pm 9.5 \mathrm{~kg}$ ) with competitive experience of at least three years and able to participate without any physical limitation.

Interventions (or Assessment of Risk Factors) Pre-practice heart rate variability (HRV), energy level, level of soreness, and hours of sleep were recorded before every practice. The number of jumps, the activity minutes, post-practice rating of perceived exertion (RPE), and HRV value the morning after were also collected. Day of the week, previous strength and conditioning practice, quality of sleep, and medical/physio attention were additional factors included in the analyses. 
Main Outcome Measurements Fatigue expressed as the percentage of jump-loss (10\%) was the dependent binary variable. A stepwise logistic regression analysis was used to analyze the relationship between fatigue, covariates, and factors.

Results Previous soreness and the number of jumps performed in practice or competition were the only factors found to be related to a significant level of fatigue experienced by the athletes $(\mathrm{p}<0.001)$.

Conclusions Although monitoring processes in team sports are today frequent, not all the load markers seem to have the same importance explaining the level of fatigue experienced by the athletes. Pre-practice level of muscle soreness and the number of jumps performed during the activity, a specific expression of external load in volleyball, reveal as the key elements to be controlled by coaches and practitioners to promote an optimal load adaptation.

\section{ABSTRACT WITHDRAWN}

\section{PERCEPTIONS OF TRAINING LOAD AND WELLNESS MONITORING OF STELLENBOSCH UNIVERSITY HIGH PERFORMANCE STUDENT-ATHLETES}

1,2 Lindsay Starling, ${ }^{3}$ Grant Van Velden, ${ }^{1,2,3}$ Sean Surmon, 1,2 Wayne Derman, 1,2 James Craig Brown. 'Institute of Sport and Exercise Medicine, Division of Orthopaedics, Department of Surgical Sciences, Faculty of Medicine and Health Sciences, Stellenbosch University, Cape Town, South Africa; ${ }^{2}$ IOC Research Centre, Cape Town, South Africa; ${ }^{3}$ Maties Sport, Stellenbosch University, Stellenbosch, South Africa

\subsection{6/bjsports-2021-IOC. 109}

Background The effective monitoring of athletes can assist in optimising their performance. This monitoring is particularly important in university student-athletes who have academic stressors additional to their training. The Stellenbosch University High Performance programme manages the top studentathletes and have implemented a training load and wellness monitoring system to assist with this.

Objective The aim of this study was to investigate the studentathletes' perceptions of this monitoring system and identify potential barriers to their adherence to the programme.

Design Cross-sectional survey.

Setting Students (young adults) who were part of the Stellenbosch University High Performance programme in 2019.

Patients (or Participants) All 156 High Performance athletes across six sporting codes received the survey, of which 146 (96\%) submitted a complete survey.

Interventions (or Assessment of Risk Factors) A six-question survey was distributed via the programme manager to the student-athletes $(n=156)$.

Main Outcome Measurements Four of this survey's questions were based upon a study conducted in nine elite U.K. athletes and two additional questions were specific to the Stellenbosch High Performance context. Results were presented as frequencies on the original studies Likert scale.

Results Half $(50 \%, \mathrm{n}=74)$ of all athletes agreed that they received sufficient feedback from the data that they entered. Almost half $(46 \%, n=69)$ agreed that sufficient action was taken by their Strength and Conditioning trainers when they indicated a meaningful change in their monitoring scores. Almost all $(97 \%, n=144)$ athletes agreed that they responded honestly to training monitoring questions. About two-thirds $(67 \%, n=100)$ of athletes agreed that training monitoring and feedback helped to optimise their training performances.

Conclusions The Stellenbosch High Performance student-athletes were substantially more positive about training monitoring than the elite UK athletes. This positivity bodes well for the Stellenbosch High Performance programme, but also highlights the importance of regular feedback to these studentathletes.

\section{APPLICATION OF THE ACUTE:CHRONIC WORKLOAD RATIO IN CHILDREN}

${ }^{1,3}$ Chinchin Wang, ${ }^{2}$ Tyrel Stokes, ${ }^{2}$ Russell Steele, ${ }^{1,3}$ lan Shrier. ${ }^{2}$ Department of Epidemiology, Biostatistics and Occupational Health, McGill University, Montreal, Canada; ${ }^{2}$ Department of Mathematics and Statistics, McGill University, Montreal, Canada; ${ }^{3}$ Centre for Clinical Epidemiology, Lady Davis Institute for Medical Research, Montreal, Canada

\subsection{6/bjsports-2021-IOC.110}

Background The IOC recommends using the acute:chronic workload ratio (ACRatio) to quantify changes in relative activity. The ACRatio has been used in adult and youth but not in children.

Objective Determine the relationship between the ACRatio and new onset pain in children.
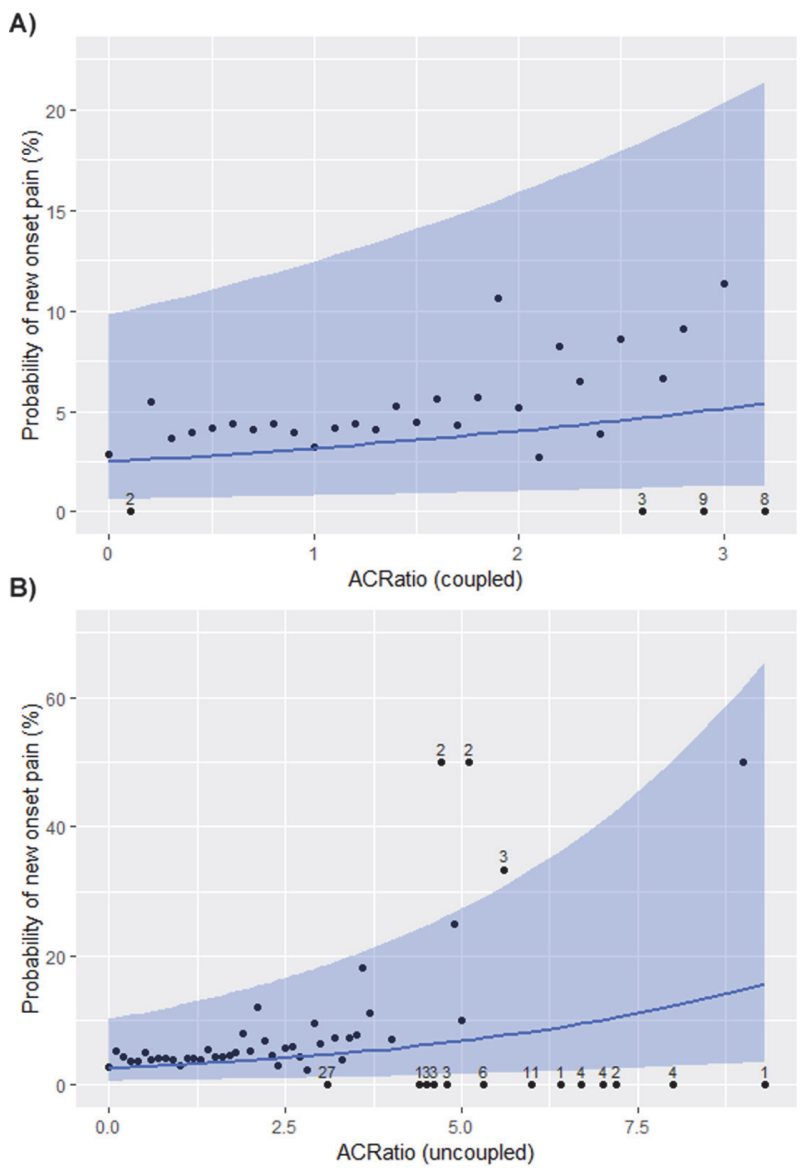

Abstract 118 Figure 1 Probability of new onset pain at different A) coupled and B) uncoupled ACRatios. Logistic regression curves with $95 \% \mathrm{Cls}$ were fitted. The number of observations are shown for outliers 